\title{
A Special Class of Infinite Dimensional Dirac Operators on the Abstract Boson-Fermion Fock Space
}

\author{
Asao Arai \\ Department of Mathematics, Hokkaido University, Sapporo 060-0810, Japan \\ Correspondence should be addressed to Asao Arai; arai@math.sci.hokudai.ac.jp
}

Received 31 May 2014; Accepted 18 August 2014; Published 8 September 2014

Academic Editor: Nasser Saad

Copyright (C) 2014 Asao Arai. This is an open access article distributed under the Creative Commons Attribution License, which permits unrestricted use, distribution, and reproduction in any medium, provided the original work is properly cited.

\begin{abstract}
Spectral properties of a special class of infinite dimensional Dirac operators $Q(\alpha)$ on the abstract boson-fermion Fock space $\mathscr{F}(\mathscr{H}, \mathscr{K})$ associated with the pair $(\mathscr{H}, \mathscr{K})$ of complex Hilbert spaces are investigated, where $\alpha \in \mathbb{C}$ is a perturbation parameter (a coupling constant in the context of physics) and the unperturbed operator $Q(0)$ is taken to be a free infinite dimensional Dirac operator. A variety of the kernel of $Q(\alpha)$ is shown. It is proved that there are cases where, for all sufficiently large $|\alpha|$ with $\alpha<0$, $Q(\alpha)$ has infinitely many nonzero eigenvalues even if $Q(0)$ has no nonzero eigenvalues. Also Fredholm property of $Q(\alpha)$ restricted to a subspace of $\mathscr{F}(\mathscr{H}, \mathscr{K})$ is discussed.
\end{abstract}

\section{Introduction}

In a previous paper [1] (cf. [2]), the author introduced a general class of infinite dimensional Dirac type operators $Q_{A}$ on the abstract boson-fermion Fock space $\mathscr{F}(\mathscr{H}, \mathscr{K})$ associated with the pair $(\mathscr{H}, \mathscr{K})$ of complex Hilbert spaces (for the definition of $\mathscr{F}(\mathscr{H}, \mathscr{K})$ and $Q_{A}$, see Section 2), where $A$ is a densely defined closed linear operator from $\mathscr{H}$ to $\mathscr{K}$. The operator $Q_{A}$ gives an infinite dimensional and abstract version of finite dimensional Dirac type operators. In applications to physics, $Q_{A}$ unifies self-adjoint supercharges (generators of supersymmetry) of some supersymmetric quantum field models (e.g., [3-6]).

In the paper [1], basic properties of $Q_{A}$ are discussed. As for the spectral properties of $Q_{A}$, only the zero-eigenvalue of $Q_{A}$ is considered. Moreover, a class of perturbations $V$ for $Q_{A}$ is introduced and, under a suitable condition, a path (functional) integral representation for the index of the perturbed operator $Q_{A}(V):=Q_{A}+V$ restricted to a subspace of $\mathscr{F}(\mathscr{H}, \mathscr{K})$ is established. The (essential) self-adjointness of $Q_{A}(V)$ is partially discussed in [7]. Analysis of other properties of $Q_{A}(V)$ including spectral ones except for the zero-eigenvalue has been left open.

In this paper, we undertake a comprehensive operatortheoretical analysis for $Q_{A}(V)$. But, as a preliminary, we consider, in the present paper, only the case where $V$ is a simple form and see what kind of phenomena occurs under the perturbation $V$.

The outline of the present paper is as follows. In Section 2 we review briefly some contents in [1]. In Section 3 we identify the spectra of $Q_{A}$. This is done via spectral analysis of a second quantization operator on the boson-fermion Fock space $\mathscr{F}(\mathscr{H}, \mathscr{K})$. In Section 4 , we introduce a perturbation $V=V_{g, v}(\alpha)$ with $g \in \mathscr{H} \backslash\{0\}$ and $v \in \mathscr{K} \backslash\{0\}$, where $\alpha \in \mathbb{C}$ is a perturbation parameter (a coupling constant in the context of physics). The perturbed operator $Q(\alpha):=Q_{A}+$ $V_{g, v}(\alpha)$ is the main object of our analysis in the present paper. We prove the self-adjointness of $Q(\alpha)$ and the essential selfadjointness of it on a suitable dense subspace of $\mathscr{F}(\mathscr{H}, \mathscr{K})$ with some other properties (Theorem 14). Also the spectra of $Q(\alpha)$ are identified (Theorem 16). Moreover, it is shown that the domain of $Q(\alpha)$ is equal to that of $Q(0)=Q_{A}$ for all sufficiently small $|\alpha|$ (Theorem 17). Section 5 is devoted to analysis of the kernel of $Q(\alpha)$. We see that the kernel property of $Q(\alpha)$ may be sensitive to conditions for $\left(A, A^{*}, \alpha\right)$. In Section 6 we consider Fredholm property of $Q(\alpha)_{+}$, the restriction of $Q(\alpha)$ to a subspace of $\mathscr{F}(\mathscr{H}, \mathscr{K})$, in comparison with that of $\left(Q_{A}\right)_{+}=Q(0)_{+}$. We obtain some classification on Fredholm property of $Q(\alpha)_{+}$(Theorem 26). An interesting phenomenon occurs in the following sense: for a constant $\alpha_{0} \neq 0$ (resp., $\beta_{0} \neq 0$ ), $Q\left(\alpha_{0}\right)_{+}$is not semi-Fredholm even if $\left(Q_{A}\right)_{+}$is Fredholm (resp., semi-Fredholm). In the last 
section, we consider nonzero eigenvalues of $Q(\alpha)$. We show that, under a suitable condition, for all sufficiently large $|\alpha|$ with $\alpha<0, Q(\alpha)$ has infinitely many nonzero eigenvalues even if $Q_{A}$ has no nonzero eigenvalues (Theorem 27). This structure also is interesting, which may be regarded as a strong coupling effect.

\section{Brief Review on the Theory of Dirac Operators on the Abstract Boson-Fermion Fock Space}

2.1. Definitions. For a linear operator $L$ on a Hilbert space, we use the following notation:

(i) $D(L)$ : the domain of $L$,

(ii) $R(L)$ : the range of $L$,

(iii) $\operatorname{ker} L:=\{\psi \in D(L) \mid L \psi=0\}$ : the kernel of $L$,

(iv) $\rho(L) \subset \mathbb{C}$ : the resolvent set of $L(\lambda \in \rho(L) \Leftrightarrow L$ is injective with $R(L)$ being dense and the inverse operator $(L-\lambda)^{-1}$ is bounded),

(v) $\sigma(L):=\mathbb{C} \backslash \rho(L)$ : the spectrum of $L$,

(vi) $\sigma_{p}(L)$ : the point spectrum of $L$ (the set of eigenvalues of $L)$.

For a complex Hilbert space $\mathscr{H}$ and a nonnegative integer $n$, we denote by $\otimes_{s}^{n} \mathscr{H}$ (resp., $\left.\wedge^{n}(\mathscr{H})\right)$ the $n$-fold symmetric (resp., antisymmetric) tensor product Hilbert space of $\mathscr{H}$ with convention $\otimes_{s}^{0} \mathscr{H}:=\mathbb{C}$ (the complex number field) and $\wedge^{0}(\mathscr{H}):=\mathbb{C}$. In terms of these Hilbert spaces, one can construct two types of larger Hilbert spaces. The one is the boson (symmetric) Fock space over $\mathscr{H}$, denoted by $\mathscr{F}_{b}(\mathscr{H})$, which is defined to be the complete infinite direct sum of $\otimes_{s}^{n} \mathscr{H}, n \geq 0$ :

$$
\begin{aligned}
& \mathscr{F}_{b}(\mathscr{H}) \\
& :=\oplus_{n=0}^{\infty} \otimes_{s}^{n} \mathscr{H} \\
& =\left\{\psi=\left\{\psi^{(n)}\right\}_{n=0}^{\infty} \mid \psi^{(n)} \in \otimes_{s}^{n} \mathscr{H}, n \geq 0, \sum_{n=0}^{\infty}\left\|\psi^{(n)}\right\|^{2}<\infty\right\},
\end{aligned}
$$

where, for a complex Hilbert space $\mathscr{X}$, we denote its inner product (resp., norm) by $\langle\cdot, \cdot\rangle$ (linear in the second variable) (resp., $\|\cdot\|)$. The other is the fermion (antisymmetric) Fock space over $\mathscr{H}$, denoted by $\mathscr{F}_{f}(\mathscr{H})$, which is defined to be the complete infinite direct sum of $\wedge^{n}(\mathscr{H}), n \geq 0$ :

$$
\begin{aligned}
& \mathscr{F}_{f}(\mathscr{H}) \\
& :=\oplus_{n=0}^{\infty} \wedge^{n}(\mathscr{H}) \\
& =\left\{\psi=\left\{\psi^{(n)}\right\}_{n=0}^{\infty} \mid \psi^{(n)} \in \wedge^{n}(\mathscr{H}), n \geq 0, \sum_{n=0}^{\infty}\left\|\psi^{(n)}\right\|^{2}<\infty\right\} .
\end{aligned}
$$

For general theories of boson and fermion Fock spaces, see, for example, [8-10].

Let $\mathscr{K}$ be a complex Hilbert space. Then the abstract boson-fermion Fock space associated with the pair $(\mathscr{H}, \mathscr{K})$ is defined as the tensor product Hilbert space

$$
\mathscr{F}(\mathscr{H}, \mathscr{K}):=\mathscr{F}_{b}(\mathscr{H}) \otimes \mathscr{F}_{f}(\mathscr{K})
$$

of $\mathscr{F}_{b}(\mathscr{H})$ and $\mathscr{F}_{f}(\mathscr{K})$.

For each $f \in \mathscr{H}$, we denote by $a(f)$ the bosonic annihilation operator on $\mathscr{F}_{b}(\mathscr{H})$ with test vector $f$; that is, $a(f)$ is the densely defined closed linear operator acting in $\mathscr{F}_{b}(\mathscr{H})$ such that its adjoint $a(f)^{*}$ is given by the following form:

$$
\begin{array}{r}
\left(a(f)^{*} \psi\right)^{(0)}=0, \quad\left(a(f)^{*} \psi\right)^{(n)}=\sqrt{n} S_{n}\left(f \otimes \psi^{(n-1)}\right), \\
n \geq 1, \quad \psi \in D\left(a(f)^{*}\right),
\end{array}
$$

where $S_{n}$ is the symmetrization operator acting on the $n$-fold tensor product Hilbert space $\otimes^{n} \mathscr{H}$.

Let

$$
\Omega_{b}:=\{1,0,0, \ldots\} \in \mathscr{F}_{b}(\mathscr{H}),
$$

called the bosonic Fock vacuum. Then, for all $f \in \mathscr{H}$,

$$
a(f) \Omega_{b}=0 .
$$

For each $u \in \mathscr{K}$, there exists a unique linear operator $b(u)$ on $\mathscr{F}_{f}(\mathscr{K})$, called the fermionic annihilation operator with test vector $u$, which has the following properties: (i) $D(b(u))=\mathscr{F}_{f}(\mathscr{K})$ and $b(u)$ is bounded with operator norm $\|b(u)\|=\|u\|$; (ii) its adjoint $b(u)^{*}$ is of the following form:

$$
\begin{aligned}
\left(b(u)^{*} \psi\right)^{(0)}=0, \quad\left(b(u)^{*} \psi\right)^{(n)} & =\sqrt{n} A_{n}\left(u \otimes \psi^{(n-1)}\right), \\
n & \geq 1, \quad \psi \in \mathscr{F}_{f}(\mathscr{K}),
\end{aligned}
$$

where $A_{n}$ is the antisymmetrization operator acting on the $n$-fold tensor product Hilbert space $\otimes^{n} \mathscr{H}$. The following canonical anticommutation relations (CAR) hold:

$$
\left\{b(u), b(v)^{*}\right\}=\langle u, v\rangle, \quad\{b(u), b(v)\}=0, \quad u, v \in \mathscr{K},
$$

where $\{X, Y\}:=X Y+Y X$. In particular, one has

$$
b(u)^{2}=0, \quad u \in \mathscr{K} .
$$

The vector

$$
\Omega_{f}:=\{1,0,0, \ldots\} \in \mathscr{F}_{f}(\mathscr{K})
$$


is called the fermionic Fock vacuum and obeys

$$
b(u) \Omega_{f}=0, \quad u \in \mathscr{K} .
$$

2.2. Infinite Dimensional Exterior Differential Operators. For a subset $\mathscr{D}$ of a Hilbert space, we denote by $\mathscr{L}(\mathscr{D})$ the subspace algebraically spanned by all the vectors of $\mathscr{D}$.

Let $A$ be a densely defined closed linear operator from $\mathscr{H}$ to $\mathscr{K}$ and define

$$
\begin{aligned}
\mathscr{D}_{A}^{\infty}:=\mathscr{L}\left(\left\{a\left(f_{1}\right)^{*} \cdots a\left(f_{n}\right)^{*} \Omega_{b} \otimes b\left(u_{1}\right)^{*}\right.\right. & \\
& \cdots b\left(u_{p}\right)^{*} \Omega_{f} \mid n, p \geq 0, f_{j} \in C^{\infty}\left(A^{*} A\right), \\
& \left.\left.j=1, \ldots, n, u_{k} \in C^{\infty}\left(A A^{*}\right), k=1, \ldots, p\right\}\right),
\end{aligned}
$$

where $C^{\infty}(L):=\cap_{n=1}^{\infty} D\left(L^{n}\right)$ for a linear operator $L$ on a Hilbert space (the symbol $X_{1} \cdots X_{n} \Omega$ with $n=0$ should read $\Omega)$. It follows that $\mathscr{D}_{A}^{\infty}$ is dense in $\mathscr{F}(\mathscr{H}, \mathscr{K})$.

The following proposition is proved in [1].

Proposition 1. There exists a unique densely defined closed linear operator $d_{A}$ on $\mathscr{F}(\mathscr{H}, \mathscr{K})$ such that the following (i) and (ii) hold:

(i) $\mathscr{D}_{A}^{\infty} \subset D\left(d_{A}\right)$ and $\mathscr{D}_{A}^{\infty}$ is a core of $d_{A}$.

(ii) For each vector $\Psi \in \mathscr{D}_{A}^{\infty}$ of the form

$$
\Psi=a\left(f_{1}\right)^{*} \cdots a\left(f_{n}\right)^{*} \Omega_{b} \otimes b\left(u_{1}\right)^{*} \cdots b\left(u_{p}\right)^{*} \Omega_{f},
$$

$d_{A}$ acts as

$$
\begin{gathered}
d_{A} \Psi=0 \quad \text { for } n=0 \\
d_{A} \Psi=\sum_{j=1}^{n} a\left(f_{1}\right)^{*} \cdots \widehat{a\left(f_{j}\right)^{*}} \cdots a\left(f_{n}\right)^{*} \Omega_{b} \\
\otimes b\left(A f_{j}\right)^{*} b\left(u_{1}\right)^{*} \cdots b\left(u_{p}\right)^{*} \Omega_{f} \quad \text { for } n \geq 1,
\end{gathered}
$$

where $\widehat{a\left(f_{j}\right)^{*}}$ indicates the omission of $a\left(f_{j}\right)^{*}$. In particular, $d_{A}$ leaves $\mathscr{D}_{A}^{\infty}$ invariant.

Moreover, the following (iii)-(v) hold:

(iii) $\mathscr{D}_{A}^{\infty} \subset D\left(d_{A}^{*}\right)$ and

$$
\begin{gathered}
d_{A}^{*} \Psi=\sum_{k=1}^{p}(-1)^{k-1} a\left(A^{*} u_{k}\right)^{*} a\left(f_{1}\right)^{*} \cdots a\left(f_{n}\right)^{*} \Omega_{b} \\
\otimes b\left(u_{1}\right)^{*} \cdots \widehat{b\left(u_{k}\right)^{*}} \cdots b\left(u_{p}\right)^{*} \Omega_{f}
\end{gathered}
$$

for all vectors $\Psi$ of the form (13) with $p \geq 1$. In the case $p=0$, we have $d_{A}^{*} \Psi=0$. In particular, $d_{A}^{*}$ leaves $\mathscr{D}_{A}^{\infty}$ invariant. (iv) $D\left(d_{A}^{2}\right)=D\left(d_{A}\right)$ and, for all $\Psi \in D\left(d_{A}\right), d_{A}^{2} \Psi=0$.

(v) Let $B$ be a bounded linear operator from $\mathscr{H}$ to $\mathscr{K}$ with $D(B)=\mathscr{H}$. Then, for all $\Psi \in \mathscr{D}_{A}^{\infty}$ and $\alpha, \beta \in \mathbb{C}$,

$$
\alpha d_{A} \Psi+\beta d_{B} \Psi=d_{\alpha A+\beta B} \Psi
$$

The operator $d_{A}$ is an abstract version of finite dimensional exterior differential operators, including an infinite dimensional version of them if $\mathscr{H}$ and $\mathscr{K}$ are infinite dimensional.

2.3. Infinite Dimensional Dirac Operators. Using the operator $d_{A}$, we define a free Dirac operator on $\mathscr{F}(\mathscr{H}, \mathscr{K})$ by

$$
Q_{A}:=d_{A}+d_{A}^{*}
$$

with $D\left(Q_{A}\right)=D\left(d_{A}\right) \cap D\left(d_{A}^{*}\right)$. We set

$$
\Delta_{A}:=d_{A}^{*} d_{A}+d_{A} d_{A}^{*},
$$

a Laplace-Beltrami operator on $\mathscr{F}(\mathscr{H}, \mathscr{K})$.

For a self-adjoint operator $S$ on $\mathscr{H}$, we define an operator $d \Gamma_{b}^{(n)}(S)$ on $\otimes_{s}^{n} \mathscr{H}$ by

$$
d \Gamma_{b}^{(n)}(S):=\frac{d \Gamma_{b}^{(0)}(S):=0,}{\sum_{j=1}^{n} I \otimes \cdots \otimes I \otimes \stackrel{j \text { th }}{S} \otimes I \otimes \cdots \otimes I, \quad n \geq 1,}
$$

where $I$ denotes identity and, for a closable linear operator $C, \bar{C}$ denotes the closure of $C$. For each $n \geq 0, d \Gamma_{b}^{(n)}(S)$ is self-adjoint. The second quantization $d \Gamma_{b}(S)$ of $S$ on $\mathscr{F}_{b}(\mathscr{H})$ is defined by

$$
d \Gamma_{b}(S):=\oplus_{n=0}^{\infty} d \Gamma_{b}^{(n)}(S),
$$

which is self-adjoint (see, e.g., [9, Section 5.2], [11, p.302, Example 2]). It follows that

$$
0 \in \sigma_{p}\left(d \Gamma_{b}(S)\right), \quad \Omega_{b} \in \operatorname{ker}\left(d \Gamma_{b}(S)\right)
$$

Similarly, for a self-adjoint operator $T$ on $\mathscr{K}$, we can define the second quantization $d \Gamma_{f}(T)$ of $T$ on $\mathscr{F}_{f}(\mathscr{K})$ by

$$
d \Gamma_{f}(T):=\oplus_{p=0}^{\infty} d \Gamma_{f}^{(p)}(T)
$$

where

$$
d \Gamma_{f}^{(p)}(T):=\frac{d \Gamma_{f}^{(0)}(T):=0,}{\sum_{j=1}^{p} I \otimes \cdots \otimes I \otimes \stackrel{j}{T}_{T}^{\text {th }} \otimes I \otimes \cdots \otimes I,} \quad p \geq 1,
$$


acting in $\wedge^{p}(\mathscr{K})$. Similarly to the case of $d \Gamma_{b}(S)$, one has

$$
0 \in \sigma_{p}\left(d \Gamma_{f}(T)\right), \quad \Omega_{f} \in \operatorname{ker}\left(d \Gamma_{f}(T)\right) .
$$

The number operator on $\mathscr{F}_{f}(\mathscr{K})$ is defined by

$$
N_{f}:=d \Gamma_{f}(I) .
$$

Then the operator

$$
\Gamma:=I \otimes(-1)^{N_{f}}
$$

on $\mathscr{F}(\mathscr{H}, \mathscr{K})$ is self-adjoint with $\Gamma^{2}=I$. The spectrum of $\Gamma$ is $\{ \pm 1\}$ with eigenspaces

$$
\begin{gathered}
\mathscr{F}_{+}(\mathscr{H}, \mathscr{K}):=\oplus_{p=0}^{\infty} \mathscr{F}_{b}(\mathscr{H}) \otimes \wedge^{2 p}(\mathscr{K}), \\
\mathscr{F}_{-}(\mathscr{H}, \mathscr{K}):=\oplus_{p=0}^{\infty} \mathscr{F}_{b}(\mathscr{H}) \otimes \wedge^{2 p+1}(\mathscr{K}),
\end{gathered}
$$

giving the orthogonal decomposition

$$
\mathscr{F}(\mathscr{H}, \mathscr{K})=\mathscr{F}_{+}(\mathscr{H}, \mathscr{K}) \oplus \mathscr{F}_{-}(\mathscr{H}, \mathscr{K}) .
$$

Let $P_{+}$and $P_{-}$be the orthogonal projections onto $\mathscr{F}_{+}(\mathscr{H}, \mathscr{K})$ and $\mathscr{F}_{-}(\mathscr{H}, \mathscr{K})$, respectively. Then

$$
\Gamma=P_{+}-P_{-} .
$$

For notational simplicity we set

$$
H_{b}(S):=d \Gamma_{b}(S), \quad H_{f}(T):=d \Gamma_{f}(T) .
$$

We introduce an operator

$$
H_{B F}(A):=H_{b}\left(A^{*} A\right) \otimes I+I \otimes H_{f}\left(A A^{*}\right)
$$

acting in $\mathscr{F}(\mathscr{H}, \mathscr{K})$, which is nonnegative and self-adjoint. It follows from (21) and (24) that

$$
0 \in \sigma_{p}\left(H_{B F}\right), \quad \Omega_{b} \otimes \Omega_{f} \in \operatorname{ker}\left(H_{B F}(A)\right) .
$$

Theorem 2 (see [1]). (i) The operator $Q_{A}$ is self-adjoint and essentially self-adjoint on every core of $H_{B F}(A)$. In particular, $Q_{A}$ is essentially self-adjoint on $\mathscr{D}_{A}^{\infty}$.

(ii) The operator $\Gamma$ leaves $D\left(Q_{A}\right)$ invariant and

$$
\Gamma Q_{A}+Q_{A} \Gamma=0
$$

on $D\left(Q_{A}\right)$.

(iii) The following operator equalities hold:

$$
\Delta_{A}=H_{B F}(A)=Q_{A}^{2} .
$$

Remark 3. The quadruple $\left\{\mathscr{F}(\mathscr{H}, \mathscr{K}), Q_{A}, H_{B F}(A), \Gamma\right\}$ is an abstract form of models of free supersymmetric quantum field theory, where $Q_{A}, H_{B F}(A)$, and $\Gamma$ are a self-adjoint supercharge, a supersymmetric Hamiltonian, and a sign operator of states (a "fermion number operator"), respectively [1].

For a linear operator $L$, we set

$$
\operatorname{nul} L:=\operatorname{dim} \operatorname{ker} L
$$

the nullity of $L$.
Theorem 4 (see [1, Lemma 3.4]).

$$
\operatorname{ker} Q_{A}=\operatorname{ker} H_{B F}(A)=\oplus_{n, p=0}^{\infty}\left[\left(\otimes_{s}^{n} \operatorname{ker} A\right) \otimes \wedge^{p}\left(\operatorname{ker} A^{*}\right)\right] .
$$

Hence the following hold.

(i) Let nul $A=0$ and $\operatorname{nul} A^{*}<\infty$. Then nul $Q_{A}=$ $2^{\text {nul } A^{*}}<\infty$.

(ii) Let nul $A=0$ and nul $A^{*}=\infty$. Then nul $Q_{A}=\infty$.

(iii) Let nul $A \geq 1$. Then nul $Q_{A}=\infty$.

2.4. Fredholm Property and Index. Let $Q$ be a self-adjoint operator on $\mathscr{F}(\mathscr{H}, \mathscr{K})$ such that $\Gamma$ leaves $D(Q)$ invariant satisfying $\Gamma Q+Q \Gamma=0$ on $D(Q)$. This class of operators $Q$ may be an abstract category of Dirac operators on the abstract boson-fermion Fock space $\mathscr{F}(\mathscr{H}, \mathscr{K})$.

Then it is easy to show that the operator $Q_{+}$defined by

$$
\begin{gathered}
D\left(Q_{+}\right):=D(Q) \cap \mathscr{F}_{+}(\mathscr{H}, \mathscr{K}), \\
Q_{+} \Psi:=Q \Psi, \quad \Psi \in D\left(Q_{+}\right),
\end{gathered}
$$

is a densely defined closed linear operator from $\mathscr{F}_{+}(\mathscr{H}, \mathscr{K})$ to $\mathscr{F}_{-}(\mathscr{H}, \mathscr{K})$. We define an index $\operatorname{ind}_{\Gamma} Q$ of $Q$ by

$$
\operatorname{ind}_{\Gamma} Q:=\operatorname{nul}_{+}-\operatorname{nul} Q_{+}^{*} \text {, }
$$

provided that at least one of nul $Q_{+}$and nul $Q_{+}^{*}$ is finite. Note that

$$
\operatorname{ind}_{\Gamma} Q=\operatorname{ind} Q_{+} \text {, }
$$

the index of $Q_{+}$(e.g., [12, Chapter IV, Section 5]).

As for the Fredholm property of $Q_{A}$, one has the following theorem.

Theorem 5 (see [1, Proposition 3.2]). (i) Let A be injective and Fredholm. Then $\left(Q_{A}\right)_{+}$is Fredholm with

$$
\operatorname{ind}_{\Gamma} Q_{A}=\delta_{0, \operatorname{nul} A^{*}} .
$$

(ii) Let $A$ be semi-Fredholm with nul $A \geq 1$ and let $A^{*}$ be injective. Then $\left(Q_{A}\right)_{+}$is semi-Fredholm with

$$
\operatorname{ind}_{\Gamma} Q_{A}=\infty \text {. }
$$

(iii) Let $A$ be semi-Fredholm with nul $A \geq 1$ and nul $A^{*} \geq$ 1. Then $\left(Q_{A}\right)_{+}$is not semi-Fredholm.

\section{Spectra of $H_{B F}(A)$ and $Q_{A}$}

Now we go into analyzing new aspects in the operator theory of $Q_{A}$ and its perturbations. In the previous paper [1], the identification of the spectra of $H_{B F}(A)$ and $Q_{A}$ was not discussed except for zero-eigenvalues. This section is devoted to identifying completely the spectra of $H_{B F}(A)$ and $Q_{A}$.

In what follows, we assume that $\mathscr{H}$ and $\mathscr{K}$ are separable.

We say that a subset $J$ of $\mathbb{R}$ is symmetric with respect to the origin if $\lambda \in J$ implies that $-\lambda \in J$. 
The main results in this section are as follows.

\section{Theorem 6.}

$$
\begin{aligned}
& \sigma\left(H_{B F}(A)\right) \\
& =\{0\} \cup \overline{\left(\bigcup_{n=1}^{\infty}\left\{\sum_{j=1}^{n} \lambda_{j} \mid \lambda_{j} \in \sigma\left(A^{*} A\right) \backslash\{0\}, j=1, \ldots, n\right\}\right)},
\end{aligned}
$$

$$
\begin{gathered}
\sigma_{p}\left(H_{B F}(A)\right) \\
=\{0\} \cup\left(\bigcup _ { n = 1 } ^ { \infty } \left\{\sum_{j=1}^{n} \lambda_{j} \mid \lambda_{j} \in \sigma_{p}\left(A^{*} A\right) \backslash\{0\},\right.\right. \\
j=1, \ldots, n\}) .
\end{gathered}
$$

Theorem 7. The spectrum $\sigma\left(Q_{A}\right)$ and the point spectrum $\sigma_{p}\left(Q_{A}\right)$ of $Q_{A}$ are symmetric with respect to the origin and

$$
\begin{aligned}
& \sigma\left(Q_{A}\right)=\{0\} \cup\left(\bigcup_{n=1}^{\infty}\left\{ \pm \sqrt{\sum_{j=1}^{n} \lambda_{j} \mid} \lambda_{j} \in \sigma\left(A^{*} A\right) \backslash\{0\}, j=1, \ldots, n\right\}\right) \\
& \sigma_{p}\left(Q_{A}\right)=\{0\} \cup\left(\bigcup_{n=1}^{\infty}\left\{ \pm \sqrt{\left.\sum_{j=1}^{n} \lambda_{j} \mid \lambda_{j} \in \sigma_{p}\left(A^{*} A\right) \backslash\{0\}, j=1, \ldots, n\right\}}\right)\right.
\end{aligned}
$$

with

$$
\operatorname{nul}\left(Q_{A}-\lambda\right)=\operatorname{nul}\left(Q_{A}+\lambda\right), \quad \lambda \in \sigma_{p}\left(Q_{A}\right) .
$$

To prove these theorems, we need a series of lemmas.

Lemma 8. Let $S$ be a self-adjoint operator on a Hilbert space.

(i) Suppose that $\sigma(S)$ is symmetric with respect to the origin. Then

$$
\sigma(S)=\left\{ \pm \sqrt{\lambda} \mid \lambda \in \sigma\left(S^{2}\right)\right\}
$$

(ii) Suppose that $\sigma_{p}(S)$ is symmetric with respect to the origin. Then

$$
\sigma_{p}(S)=\left\{ \pm \sqrt{\lambda} \mid \lambda \in \sigma_{p}\left(S^{2}\right)\right\} .
$$

Moreover, for each $f \in \operatorname{ker}\left(S^{2}-\lambda\right)$ with $\lambda \in \sigma_{p}\left(S^{2}\right)$, $(S \pm \sqrt{\lambda}) f \in \operatorname{ker}(S \mp \sqrt{\lambda})$.

Proof. (i) Let $\Sigma_{S}$ be the set on the right hand side of (46). Then, by the spectral mapping theorem, we have $\sigma(S) \subset \Sigma_{S}$. Conversely, let $\mu \in \Sigma_{S}$, so that $\mu^{2} \in \sigma\left(S^{2}\right)$. Hence there exists a sequence $\left\{f_{n}\right\}_{n=1}^{\infty} \subset D\left(S^{2}\right)$ such that $\left\|f_{n}\right\|=1$ and $\left(S^{2}-\right.$ $\left.\mu^{2}\right) f_{n} \rightarrow 0(n \rightarrow \infty)$. Suppose that $\mu \notin \sigma(S)$. Then $S-\mu$ is bijective with $(S-\mu)^{-1}$ bounded. Hence $(S-\mu)^{-1}\left(S^{2}-\mu^{2}\right) f_{n} \rightarrow$ $0(n \rightarrow \infty)$, which means that $(S+\mu) f_{n} \rightarrow(n \rightarrow \infty)$. Hence $-\mu \in \sigma(S)$. Then, by the symmetry of $\sigma(S), \mu$ is in $\sigma(S)$. But this is a contradiction. Hence $\mu \in \sigma(S)$. Thus $\Sigma_{S} \subset \sigma(S)$.

(ii) Let $\Sigma_{S, p}$ be the set on the right hand side of (47). Then it is obvious that $\sigma_{p}(S) \subset \Sigma_{S, p}$. Conversely, let $\mu \in \Sigma_{S, p}$, so that $\mu^{2} \in \sigma_{p}\left(S^{2}\right)$. Hence there exists a vector $f \in D\left(S^{2}\right)$ such that $f \neq 0$ and $S^{2} f=\mu^{2} f$. The second equation is written as $(S-\mu)(S+\mu) f=0$. If $\phi:=(S+\mu) f=0$, then $-\mu \in \sigma_{p}(S)$.
Hence, by the symmetry of $\sigma_{p}(S), \mu$ is in $\sigma_{p}(S)$. If $\phi \neq 0$, then $(S-\mu) \phi=0$. Hence $\mu \in \sigma_{p}(S)$. Thus $\Sigma_{S, p} \subset \sigma_{p}(S)$. These arguments also imply the last statement.

A sufficient condition for the spectra of a self-adjoint operator to be symmetric with respect to the origin is given in the following lemma.

Lemma 9. Let $S$ be a self-adjoint operator on a Hilbert space $\mathscr{X}$. Suppose that there exists a bounded self-adjoint operator $\tau$ on $\mathscr{X}$ such that $\tau^{2}=I$ and $\tau$ leaves $D(S)$ invariant satisfying

$$
\tau S+S \tau=0 \quad \text { on } D(S) .
$$

Then $\sigma(S)$ and $\sigma_{p}(S)$ are symmetric with respect to the origin and, for all $\lambda \in \sigma_{p}(S)$,

$$
\operatorname{nul}(S-\lambda)=\operatorname{nul}(S+\lambda)
$$

Proof. Note that $\tau$ is unitary with $\tau^{-1}=\tau$. Equation (48) implies the operator equality $S=\tau(-S) \tau^{-1}$. Hence $S$ is unitarily equivalent to $-S$. Thus the desired results follow.

Lemma 10. Let $\mathscr{H}_{n}(n=0,1,2, \ldots)$ be a complex Hilbert space and $A_{n}$ be a self-adjoint operator on $\mathscr{H}_{n}$. Let $\oplus_{n=0}^{\infty} A_{n}$ be the direct sum of $A_{n}$ 's acting in the direct sum Hilbert space $\oplus_{n=0}^{\infty} \mathscr{H}_{n}$. Then, $\oplus_{n=0}^{\infty} A_{n}$ is self-adjoint and

$$
\sigma\left(\oplus_{n=0}^{\infty} A_{n}\right)=\overline{\cup_{n=0}^{\infty} \sigma\left(A_{n}\right)}, \quad \sigma_{p}\left(\oplus_{n=0}^{\infty} A_{n}\right)=\cup_{n=0}^{\infty} \sigma_{p}\left(A_{n}\right) .
$$

Proof. See, for example, [8, Proposition 3.5-(ii), Theorem 3.7(i)]. 
Lemma 11. Let $S$ be a self-adjoint operator on $\mathscr{H}$. Then

$$
\begin{aligned}
& \sigma\left(H_{b}(S)\right) \\
& \left.=\{0\} \bigcup \overline{\left(\bigcup_{n=1}^{\infty}\left\{\sum_{j=1}^{n} \lambda_{j} \mid \lambda_{j} \in \sigma(S), j=1, \ldots, n\right\}\right)}\right) \\
& \sigma_{p}\left(H_{b}(S)\right) \\
& =\{0\} \bigcup\left(\bigcup_{n=1}^{\infty}\left\{\sum_{j=1}^{n} \lambda_{j} \mid \lambda_{j} \in \sigma_{p}(S), j=1, \ldots, n\right\}\right) .
\end{aligned}
$$

Proof. The following are well known (e.g., [8, Proposition 4.12]): $\sigma\left(d \Gamma_{b}^{(0)}(S)\right)=\{0\}=\sigma_{p}\left(d \Gamma_{b}^{(0)}(S)\right)$ and, for all $n \geq 1$,

$$
\begin{gathered}
\sigma\left(d \Gamma_{b}^{(n)}(S)\right)=\overline{\left\{\sum_{j=1}^{n} \lambda_{j} \mid \lambda_{j} \in \sigma(S), j=1, \ldots, n\right\},} \\
\sigma_{p}\left(d \Gamma_{b}^{(n)}(S)\right)=\left\{\sum_{j=1}^{n} \lambda_{j} \mid \lambda_{j} \in \sigma_{p}(S), j=1, \ldots, n\right\} .
\end{gathered}
$$

By these facts and Lemma 10, we obtain (51) and (52).

For a self-adjoint operator $T$ on a Hilbert space $\mathscr{K}$ and $p=1,2, \ldots$, we define

$$
\begin{aligned}
\Sigma^{(p)}(T) & \\
:= & \left\{\sum_{j=1}^{p} \mu_{j} \mid \mu_{j} \in \sigma(T)(j=1, \ldots, p),\right. \\
& \left.\#\left\{k \mid \mu_{j}=\mu_{k}\right\} \leq \operatorname{nul}\left(T-\mu_{j}\right) \text { if } \mu_{j} \in \sigma_{d}(T)\right\},
\end{aligned}
$$

where, for a finite set $F$, \#F denotes the number of the elements of $F$ and $\sigma_{d}(T)$ denotes the discrete spectrum of $T$ (the set of isolated eigenvalues of $T$ with finite multiplicity).

Lemma 12 (see [13, Corollary 3.2]).

$$
\sigma\left(H_{f}(T)\right)=\{0\} \bigcup\left(\overline{\bigcup_{p=1}^{\infty} \Sigma^{(p)}(T)}\right),
$$

$$
\begin{gathered}
\sigma_{p}\left(H_{f}(T)\right) \\
=\{0\} \bigcup\left(\bigcup _ { p = 1 } ^ { \infty } \left\{\sum_{j=1}^{p} \mu_{j} \mid \mu_{j} \in \sigma_{p}(T),\right.\right. \\
\#\left\{k \mid \mu_{j}=\mu_{k}\right\} \leq \operatorname{nul}\left(T-\mu_{j}\right), \\
j=1, \ldots, p\}) .
\end{gathered}
$$

Proof of Theorem 6. By a theorem on the spectrum of the sum of tensor products of self-adjoint operators (e.g., [14, Corollary 7.25]),

$$
\begin{aligned}
\sigma & \left(H_{B F}(A)\right) \\
& =\left\{\lambda+\mu \mid \lambda \in \sigma\left(H_{b}\left(A^{*} A\right)\right), \mu \in \sigma\left(H_{f}\left(A A^{*}\right)\right)\right\},
\end{aligned}
$$

where we have used the fact that the set on the right hand side is closed (note that $\left.\sigma\left(H_{b}\left(A^{*} A\right)\right), \sigma\left(H_{f}\left(A A^{*}\right)\right) \subset[0, \infty)\right)$. By (51) and (55),

$$
\sigma\left(H_{B F}(A)\right) \subset\{0\} \cup \bar{\Sigma}_{1} \cup \bar{\Sigma}_{2} \cup \bar{\Sigma}_{3}
$$

with

$$
\begin{aligned}
& \Sigma_{1}:=\bigcup_{n=1}^{\infty}\left\{\sum_{j=1}^{n} \lambda_{j} \mid \lambda_{j} \in \sigma\left(A^{*} A\right), j=1, \ldots, n\right\}, \\
& \Sigma_{2}:=\bigcup_{p=1}^{\infty}\left\{\sum_{k=1}^{p} \mu_{k} \mid \mu_{k} \in \sigma\left(A A^{*}\right)(k=1, \ldots, p),\right. \\
& \#\left\{\ell \mid \mu_{k}=\mu_{\ell}\right\} \leq \operatorname{nul}\left(A A^{*}-\mu_{k}\right) \\
& \text { if } \left.\mu_{k} \in \sigma_{d}\left(A A^{*}\right)\right\} \text {, } \\
& \Sigma_{3}:=\bigcup_{n, p=1}^{\infty}\left\{\sum_{j=1}^{n} \lambda_{j}+\sum_{k=1}^{p} \mu_{k} \mid \lambda_{j} \in \sigma\left(A^{*} A\right)(j=1, \ldots, n),\right. \\
& \mu_{k} \in \sigma\left(A A^{*}\right)(k=1, \ldots, p), \\
& \#\left\{\ell \mid \mu_{k}=\mu_{\ell}\right\} \leq \operatorname{nul}\left(A A^{*}-\mu_{k}\right) \\
& \text { if } \left.\mu_{k} \in \sigma_{d}\left(A A^{*}\right)\right\} \text {. }
\end{aligned}
$$

By Deift's theorem [15], we have

$$
\sigma\left(A^{*} A\right) \backslash\{0\}=\sigma\left(A A^{*}\right) \backslash\{0\},
$$

Hence $\Sigma_{2} \subset\{0\} \cup \Sigma_{1}$ and $\Sigma_{3} \subset \Sigma_{1}$. Therefore it follows that $\sigma\left(H_{B F}(A)\right) \subset\{0\} \cup \bar{\Sigma}_{1}$. The converse inclusion relation 
$\{0\} \cup \bar{\Sigma}_{1} \subset \sigma\left(H_{B F}(A)\right)$ is obvious. Thus $\sigma\left(H_{B F}(A)\right)=\{0\} \cup \bar{\Sigma}_{1}$. The set on the right hand side is equal to the set on the right hand side of (42). Thus (42) holds.

The proof of (43) is similar to that of (42), where we use another version of Deift's theorem [15]:

$$
\sigma_{p}\left(A^{*} A\right) \backslash\{0\}=\sigma_{p}\left(A A^{*}\right) \backslash\{0\}
$$

with $\operatorname{nul}\left(A^{*} A-\lambda\right)=\operatorname{nul}\left(A A^{*}-\lambda\right)$ for each positive eigenvalue $\lambda>0$ of $A^{*} A\left(A A^{*}\right)$.

Proof of Theorem 7. By Theorem 2(ii) and Lemma 9, $\sigma\left(Q_{A}\right)$ and $\sigma_{p}\left(Q_{A}\right)$ are symmetric with respect to the origin and (45) holds. By Lemma 8 and Theorem 2(iii), we have

$$
\begin{aligned}
\sigma\left(Q_{A}\right) & =\left\{ \pm \sqrt{\lambda} \mid \lambda \in \sigma\left(H_{B F}(A)\right)\right\} \\
\sigma_{p}\left(Q_{A}\right) & =\left\{ \pm \sqrt{\lambda} \mid \lambda \in \sigma_{p}\left(H_{B F}(A)\right)\right\} .
\end{aligned}
$$

By these facts and Theorem 6, we obtain (44).

\section{A Class of Perturbed Infinite Dimensional Dirac Operators}

In this section we consider a class of perturbations of $Q_{A}$. It would be natural to perturb $Q_{A}$ through a perturbation of $d_{A}$. Let $g \in D(A) \backslash\{0\}, v \in D\left(A^{*}\right) \backslash\{0\}$ and define

$$
d(\alpha):=d_{A}+\alpha a(g) \otimes b(v)^{*},
$$

where $\alpha \in \mathbb{C}$ is a perturbation parameter (a coupling constant in the context of physics). We have by direct computation

$$
d(\alpha)^{2}=0 \quad \text { on } \mathscr{D}_{A}^{\infty} .
$$

The adjoint $d(\alpha)^{*}$ is densely defined with $\mathscr{D}_{A}^{\infty} \subset D\left(d(\alpha)^{*}\right)$ and

$$
d(\alpha)^{*}=d_{A}^{*}+\alpha^{*} a(g)^{*} \otimes b(v) \quad \text { on } \mathscr{D}_{A}^{\infty}
$$

Hecne $d(\alpha)$ is closable. We denote the closure of $d(\alpha)\left\lceil\mathscr{D}_{A}^{\infty}\right.$ by $\bar{d}(\alpha)$. Then, for all $\Psi \in D(\bar{d}(\alpha)), \bar{d}(\alpha) \Psi$ is in $D(\bar{d}(\alpha))$ and

$$
\bar{d}(\alpha)^{2} \Psi=0
$$

The operator $\bar{d}(\alpha)$ yields a perturbed Dirac operator:

$$
Q(\alpha):=\bar{d}(\alpha)+\bar{d}(\alpha)^{*} \text {. }
$$

By an application of [2, Lemma 4.1], one sees that $Q(\alpha)$ is a closed symmetric operator. We have

$$
Q(\alpha)=Q_{A}+V_{g, v}(\alpha) \quad \text { on } \mathscr{D}_{A}^{\infty}
$$

with

$$
V_{g, v}(\alpha):=\alpha a(g) \otimes b(v)^{*}+\alpha^{*} a(g)^{*} \otimes b(v) .
$$

In what follows, we investigate properties of $Q(\alpha)$.
4.1. Self-Adjointness of $Q(\alpha)$. We define a linear operator $T_{g, v}$ from $\mathscr{H}$ to $\mathscr{K}$ by

$$
T_{g, v} f:=\langle g, f\rangle v, \quad f \in \mathscr{H} .
$$

It is obvious that $T_{g, v}$ is a bounded operator (a one-rank operator). Hence

$$
A(\alpha):=A+\alpha T_{g, v}
$$

is a densely defined closed linear operator with $D(A(\alpha))=$ $D(A)$.

The following lemma is a key fact.

Lemma 13. For all $\alpha \in \mathbb{C}$, the following operator equality holds:

$$
\bar{d}(\alpha)=d_{A(\alpha)} .
$$

Proof. Let $\Psi \in \mathscr{D}_{A}^{\infty}$ be a vector of the form (13). Then, using the canonical commutation relations (CCR)

$$
\left[a(f), a(g)^{*}\right]=\langle f, g\rangle, \quad f, g \in \mathscr{H},
$$

on

$$
\begin{aligned}
& \mathscr{F}_{b \text {,fin }}(\mathscr{H}) \\
& \quad:=\mathscr{L}\left\{a\left(f_{1}\right)^{*} \cdots a\left(f_{n}\right)^{*} \Omega_{b} \mid n \geq 0, f_{j} \in \mathscr{H}, j=1, \ldots, n\right\}
\end{aligned}
$$

and (6), we have

$$
\begin{aligned}
a(g) \otimes b(v)^{*} \Psi= & \sum_{j=1}^{n}\left\langle g, f_{j}\right\rangle a\left(f_{1}\right)^{*} \cdots \overline{a\left(f_{j}\right)^{*}} \cdots a\left(f_{n}\right)^{*} \Omega_{b} \\
& \otimes b(v)^{*} b\left(u_{1}\right)^{*} \cdots b\left(u_{p}\right)^{*} \Omega_{f} \\
= & \sum_{j=1}^{n} a\left(f_{1}\right)^{*} \cdots \widehat{a\left(f_{j}\right)^{*}} \cdots a\left(f_{n}\right)^{*} \Omega_{b} \\
& \otimes b\left(T_{g, v} f_{j}\right)^{*} b\left(u_{1}\right)^{*} \cdots b\left(u_{p}\right)^{*} \Omega_{f} \\
= & d_{T_{g, v}} \Psi .
\end{aligned}
$$

Hence, by Proposition 1-(v)

$$
\bar{d}(\alpha) \Psi=d_{A} \Psi+\alpha d_{T_{g, v}} \Psi=d_{A(\alpha)} \Psi .
$$

By a limiting argument, we obtain the operator equality (72).

Theorem 14. (i) For all $\alpha \in \mathbb{C}, Q(\alpha)$ is self-adjoint and

$$
Q(\alpha)=Q_{A(\alpha)} .
$$

(ii) For all $\alpha \in \mathbb{C}, Q(\alpha)$ is essentially self-adjoint on $\mathscr{D}_{A}^{\infty}$. (iii) For all $\alpha \in \mathbb{C}$,

$$
Q(\alpha)=\overline{Q_{A}+V_{g, v}(\alpha)}
$$


(iv) The operator $\Gamma$ leaves $D(Q(\alpha))$ invariant and

$$
\Gamma Q(\alpha)+Q(\alpha) \Gamma=0 \quad \text { on } D(Q(\alpha)) \text {. }
$$

(v) For all $\Psi \in \mathscr{D}_{A}^{\infty}$, the vector-valued function: $\alpha \mapsto$ $Q(\alpha) \Psi$ is strongly continuous on $\mathbb{C}$. Moreover, for all $z \in \mathbb{C} \backslash \mathbb{R}$, $(\mathrm{Q}(\alpha)-z)^{-1}$ is strongly continuous in $\alpha \in \mathbb{C}$.

Proof. (i) Operator equality (77) follows from (72) and (67). Then, by Theorem 2-(i) with $A$ replaced by $A(\alpha)$ ), we conclude that $Q(\alpha)$ is self-adjoint.

(ii) We have the following operator equalities:

$$
\begin{aligned}
& A(\alpha)^{*} A(\alpha)=A^{*} A+X_{g, v}(\alpha), \\
& A(\alpha) A(\alpha)^{*}=A A^{*}+Y_{g, v}(\alpha),
\end{aligned}
$$

where $X_{g, v}(\alpha)$ and $Y_{g, v}(\alpha)$ are bounded linear operators on $\mathscr{H}$ and $\mathscr{K}$, respectively, given as follows:

$$
\begin{aligned}
& X_{g, v}(\alpha) f \\
& :=\alpha\langle g, f\rangle A^{*} v+\alpha^{*}\left\langle A^{*} v, f\right\rangle g+|\alpha|^{2}\|v\|^{2}\langle g, f\rangle g, \\
& f \in \mathscr{H}, \\
& Y_{g, v}(\alpha) u \\
& :=\alpha\langle A g, u\rangle v+\alpha^{*}\langle v, u\rangle A g+|\alpha|^{2}\|g\|^{2}\langle v, u\rangle v, \\
& u \in \mathscr{K} .
\end{aligned}
$$

Hence, by the Kato-Rellich theorem ([12, Chapter V, Theorems 4.3, 4.4], [10, Theorem X.12]), $A(\alpha)^{*} A(\alpha)$ (resp., $\left.A(\alpha) A(\alpha)^{*}\right)$ is essentially self-adjoint on each core of $A^{*} A$ (resp., $\left.A A^{*}\right)$. In particular, $C^{\infty}\left(A^{*} A\right)$ (resp., $C^{\infty}\left(A A^{*}\right)$ ) is a core of $A(\alpha)^{*} A(\alpha)$ (resp., $\left.A(\alpha) A(\alpha)^{*}\right)$. Hence, by a core property of second quantization operators, $\mathscr{D}_{A}^{\infty}$ is a core of $H_{B F}(A(\alpha))$. By this fact and Theorem 2-(i), $\mathscr{D}_{A}^{\infty}$ is a core of $Q_{A(\alpha)}=Q(\alpha)$.

(iii) This follows from (ii) and (68).

(iv) This follows from (77) and Theorem 2-(ii) with $A$ replaced by $A(\alpha)$.

(v) Let $\Psi \in \mathscr{D}_{A}^{\infty}$. Then, for all $\alpha, \beta \in \mathbb{C}$, we have

$$
\begin{aligned}
& \|Q(\beta) \Psi-Q(\alpha) \Psi\| \\
& \quad \leq|\beta-\alpha|\left(\left\|a(g) \otimes b(v)^{*} \Psi\right\|+\left\|a(g)^{*} \otimes b(v) \Psi\right\|\right) \\
& \quad \longrightarrow 0(\beta \longrightarrow \alpha) .
\end{aligned}
$$

Since $\mathscr{D}_{A}^{\infty}$ is a common core for $\{Q(\alpha)\}_{\alpha \in \mathbb{C}}$ as proved in part (ii), a general fact [11, Theorem VIII.25] implies the strong continuity of $(Q(\alpha)-z)^{-1}(z \in \mathbb{C} \backslash \mathbb{R})$ in $\alpha \in \mathbb{C}$.

Remark 15. Since the operators $X_{g, v}(\alpha)$ and $Y_{g, v}(\alpha)$ are finite rank, they are compact. Hence it follows from (80) and a general theorem (e.g., [16, p.113, Corollary 2]) that, for all $\alpha \in \mathbb{C}$,

$$
\begin{gathered}
\sigma_{\text {ess }}\left(A(\alpha)^{*} A(\alpha)\right)=\sigma_{\text {ess }}\left(A^{*} A\right), \\
\sigma_{\text {ess }}\left(A(\alpha) A(\alpha)^{*}\right)=\sigma_{\text {ess }}\left(A A^{*}\right),
\end{gathered}
$$

where, for a self-adjoint operator $T, \sigma_{\text {ess }}(T)$ denotes the essential spectrum of $T$. In particular, if the spectrum $\sigma\left(A^{*} A\right)$ is purely discrete (in this case, $\sigma\left(A A^{*}\right)$ also is purely discrete by $(60))$, then so are $\sigma\left(A(\alpha)^{*} A(\alpha)\right)$ and $\sigma\left(A(\alpha) A(\alpha)^{*}\right)$.

4.2. Spectra of $Q(\alpha)$. We have (77). Hence, applying Theorem 7 with $A$ replaced by $A(\alpha)$, we obtain the following result on the spectra of $Q(\alpha)$.

Theorem 16. For all $\alpha \in \mathbb{C}, \sigma(Q(\alpha))$ and $\sigma_{p}(Q(\alpha))$ are symmetric with respect to the origin and

$$
\begin{aligned}
& \sigma(Q(\alpha))=\{0\} \bigcup\left(\bigcup_{n=1}^{\infty}\left\{ \pm \sqrt{\sum_{j=1}^{n} \lambda_{j} \mid} \lambda_{j} \in \sigma\left(A(\alpha)^{*} A(\alpha)\right) \backslash\{0\}, j=1, \ldots, n\right\}\right) \\
& \sigma_{p}(Q(\alpha))=\{0\} \bigcup\left(\bigcup _ { n = 1 } ^ { \infty } \left\{ \pm \sqrt{\left.\left.\sum_{j=1}^{n} \lambda_{j} \mid \lambda_{j} \in \sigma_{p}\left(A(\alpha)^{*} A(\alpha)\right) \backslash\{0\}, j=1, \ldots, n\right\}\right)}\right.\right.
\end{aligned}
$$

with

$$
\operatorname{nul}(Q(\alpha)-\lambda)=\operatorname{nul}(Q(\alpha)+\lambda), \quad \lambda \in \sigma_{p}(Q(\alpha))
$$

This theorem shows that the spectrum and the point spectrum of $Q(\alpha)$ are completely determined from those of $A(\alpha)^{*} A(\alpha) \backslash\{0\}$.

4.3. Identification of the Domain of $Q(\alpha)$. The following theorem gives a sufficient condition for the domain of $Q(\alpha)$ to be equal to that of the unperturbed operator $Q_{A}$.
Theorem 17. Suppose that $A$ is injective and $g \in D\left(A^{-1}\right)$. Then, for all $|\alpha|<1 /\|v\|\left\|A^{-1} g\right\|, Q(\alpha)$ is self-adjoint with $D(Q(\alpha))=D\left(Q_{A}\right)$ and

$$
Q(\alpha)=Q_{A}+V_{g, v}(\alpha)
$$

Moreover, $Q(\alpha)$ is essentially self-adjoint on any core for $Q_{A}$.

To prove this theorem, we recall well known facts (e.g., [17, Lemma 2.1], [8]). 
Lemma 18. Let $S$ be a nonnegative self-adjoint operator on $\mathscr{H}$ with $\operatorname{ker} S=\{0\}$. Then, for all $f \in D\left(S^{-1 / 2}\right), D\left(d \Gamma_{b}(S)^{1 / 2}\right) \subset$ $D(a(f)) \cap D\left(a(f)^{*}\right)$ with

$$
\begin{gathered}
\|a(f) \psi\| \leq\left\|S^{-1 / 2} f\right\|\left\|d \Gamma_{b}(S)^{1 / 2} \psi\right\|, \\
\left\|a(f)^{*} \psi\right\|^{2} \leq\left\|S^{-1 / 2} f\right\|^{2}\left\|d \Gamma_{b}(S)^{1 / 2} \psi\right\|^{2}+\|f\|^{2}\|\psi\|^{2}, \\
\psi \in D\left(d \Gamma_{b}(S)^{1 / 2}\right) .
\end{gathered}
$$

Lemma 19. The operator $V_{g, v}(\alpha)$ is a closed symmetric operator with $D\left(V_{g, v}(\alpha)\right)=D\left(a(g) \otimes b(v)^{*}\right) \cap D\left(a(g)^{*} \otimes b(v)\right)$.

Proof. We need only to prove the closedness of $V_{g, v}(\alpha)$. Using (9) and a limiting argument, we have, for all $\Psi, \Phi \in$ $D\left(V_{g, v}(\alpha)\right)=D\left(a(f) \otimes b(v)^{*}\right) \cap D\left(a(f)^{*} \otimes b(v)\right)$,

$$
\left(a(g) \otimes b(v)^{*} \Psi, a(g)^{*} \otimes b(v) \Phi\right)=0 .
$$

Hence, by a general fact [2, Lemma 4.1], $V_{g, v}(\alpha)$ is closed.

Lemma 20. Let $A$ be injective and $g \in D\left(A^{-1}\right)$. Then $D\left(Q_{A}\right) \subset$ $D\left(V_{g, v}(\alpha)\right)$.

Proof. Under the present assumption, $A^{*} A$ is injective and $\left(A^{*} A\right)^{-1 / 2}=|A|^{-1}$. Let $\Psi \in \mathscr{D}_{A}^{\infty}$. Then, using (87) and the well known fact that

$$
\|b(u)\|=\left\|b(u)^{*}\right\|=\|u\|, \quad u \in \mathscr{K},
$$

we can show that

$$
\begin{aligned}
\left\|a(g) \otimes b(v)^{*} \Psi\right\| & \leq\|v\|\|a(g) \otimes I \Psi\| \\
& \leq\|v\|\left\||A|^{-1} g\right\|\left\|d \Gamma_{b}\left(A^{*} A\right)^{1 / 2} \otimes I \Psi\right\| \\
& \leq\|v\|\left\|A^{-1} g\right\|\left\|H_{B F}(A)^{1 / 2} \Psi\right\| .
\end{aligned}
$$

By this estimate and the fact that

$$
\left\|H_{B F}(A)^{1 / 2} \Phi\right\|=\left\|Q_{A} \Phi\right\|, \quad \Phi \in D\left(Q_{A}\right),
$$

we obtain

$$
\left\|a(g) \otimes b(v)^{*} \Psi\right\| \leq\|v\|\left\|A^{-1} g\right\|\left\|Q_{A} \Psi\right\| .
$$

Since $\mathscr{D}_{A}^{\infty}$ is a core of $Q_{A}$, this inequality extends to all $\Psi \in$ $D\left(Q_{A}\right)$ showing that $D\left(Q_{A}\right) \subset D\left(a(g) \otimes b(v)^{*}\right)$. Similarly, using (88), we can show that $D\left(Q_{A}\right) \subset D\left(a(g)^{*} \otimes b(v)\right)$ with

$$
\begin{array}{r}
\left\|a(g)^{*} \otimes b(v) \Psi\right\|^{2} \leq\|v\|^{2}\left(\left\|A^{-1} g\right\|^{2}\left\|Q_{A} \Psi\right\|^{2}+\|g\|^{2}\|\Psi\|^{2}\right), \\
\Psi \in D\left(Q_{A}\right) .
\end{array}
$$

Thus $D\left(Q_{A}\right) \subset D\left(V_{g, v}(\alpha)\right)$.

Proof of Theorem 17. By Lemma 20 and Theorem 14, the operator

$$
T:=Q_{A}+V_{g, v}(\alpha)
$$

is essentially self-adjoint with $D(T)=D\left(Q_{A}\right)$ and $\bar{T}=Q(\alpha)$. By (89), the CCR (73), and the CAR (8), one can show that, for all $\Psi \in D\left(Q_{A}\right)$,

$$
\begin{aligned}
& \left\|V_{g, v}(\alpha) \Psi\right\|^{2} \\
& \quad=|\alpha|^{2}\left(\left\|a(g) \otimes b(v)^{*} \Phi\right\|^{2}+\left\|a(g)^{*} \otimes b(v) \Phi\right\|^{2}\right) \\
& =|\alpha|^{2}\left(\|v\|^{2}\|a(g) \otimes I \Psi\|^{2}+\|g\|^{2}\|I \otimes b(v) \Psi\|^{2}\right) \\
& \quad \leq|\alpha|^{2}\|v\|^{2}\left(\|a(g) \otimes I \Psi\|^{2}+\|g\|^{2}\|\Psi\|^{2}\right) .
\end{aligned}
$$

Hence, using (87) (cf. the proof of Lemma 20), we obtain

$$
\begin{array}{r}
\left\|V_{g, v}(\alpha) \Psi\right\|^{2} \leq|\alpha|^{2}\|v\|^{2}\left(\left\|A^{-1} g\right\|^{2}\left\|Q_{A} \Psi\right\|^{2}+\|g\|^{2}\|\Phi\|^{2}\right), \\
\Psi \in D\left(Q_{A}\right) .
\end{array}
$$

Therefore, by the Kato-Rellich theorem, for all $\alpha \in \mathbb{C}$ satisfying $|\alpha|\|v\|\left\|A^{-1} g\right\|<1, T$ is self-adjoint on $D\left(Q_{A}\right)$, implying that $T=Q(\alpha)$, and essentially self-adjoint on any core for $Q_{A}$.

\section{Kernel of $Q(\alpha)$}

In this section we investigate the kernel of $Q(\alpha)$. For this purpose, we need some conditions on $\{A, g, v\}$.

(C.1) $A$ is injective, $v \in D\left(A^{-1}\right)$, and $\left\langle g, A^{-1} v\right\rangle \neq 0$. In this case we introduce a constant

$$
\alpha_{0}:=-\frac{1}{\left\langle g, A^{-1} v\right\rangle} .
$$

(C.2) $A^{*}$ is injective, $g \in D\left(A^{*-1}\right)$, and $\left\langle v, A^{*-1} g\right\rangle \neq$ 0 . In this case we introduce a constant

$$
\beta_{0}:=-\frac{1}{\left\langle A^{*-1} g, v\right\rangle} .
$$

(C.3) (a) $A$ is injective and $v \notin D\left(A^{-1}\right)$ or (b) $A$ is injective and $v \in D\left(A^{-1}\right)$ with $\left\langle g, A^{-1} v\right\rangle=0$.

(C.4) (a) $A^{*}$ is injective and $g \notin D\left(A^{*-1}\right)$ or (b) $A^{*}$ is injective and $g \in D\left(A^{*-1}\right)$ with $\left\langle v, A^{*-1} g\right\rangle=0$.

Remark 21. (i) If (C.1) and (C.2) hold, then it follows that $\alpha_{0}=$ $\beta_{0}$.

(ii) If $A$ is Fredholm, then $A^{*}$ is Fredholm [12, Chapter IV, Corollary 5.14]. Hence, if (C.1) and (C.2) hold and $A$ is Fredholm, then $\mathscr{H}=\overline{R\left(A^{*}\right)}=R\left(A^{*}\right), \mathscr{K}=\overline{R(A)}=$ $R(A)$. Hence $A$ and $A^{*}$ are bijective with $D\left(A^{-1}\right)=\mathscr{K}$ and $D\left(A^{*-1}\right)=\mathscr{H}$. Thus, in this case, conditions $v \in D\left(A^{-1}\right)$ and $g \in D\left(A^{*-1}\right)$ are trivially satisfied.

We first consider the kernel of $A(\alpha)$ and $A(\alpha)^{*}$. 
Lemma 22. (i) Suppose that (C.1) holds. Then

$$
\begin{gathered}
\operatorname{ker} A(\alpha)=\{0\}, \quad \alpha \neq \alpha_{0}, \\
\operatorname{ker} A\left(\alpha_{0}\right)=\left\{c A^{-1} v \mid c \in \mathbb{C}\right\} .
\end{gathered}
$$

(ii) Suppose that (C.2) holds. Then

$$
\begin{gathered}
\operatorname{ker} A(\alpha)^{*}=\{0\}, \quad \alpha \neq \beta_{0}, \\
\operatorname{ker} A\left(\beta_{0}\right)^{*}=\left\{c A^{*-1} g \mid c \in \mathbb{C}\right\} .
\end{gathered}
$$

(iii) Suppose that (C.3) holds. Then, for all $\alpha \in \mathbb{C}$,

$$
\operatorname{ker} A(\alpha)=\{0\} .
$$

(iv) Suppose that (C.4) holds. Then, for all $\alpha \in \mathbb{C}$,

$$
\operatorname{ker} A(\alpha)^{*}=\{0\} .
$$

Proof. (i) Let $f \in \operatorname{ker} A(\alpha)$. Then $f \in D(A)$ and

$$
A f=-\alpha\langle g, f\rangle \nu .
$$

Hence

$$
f=-\alpha\langle g, f\rangle f_{0}
$$

with $f_{0}:=A^{-1} v$. This implies

$$
\operatorname{ker} A(\alpha) \subset\left\{c f_{0} \mid c \in \mathbb{C}\right\} \text {. }
$$

Let $\alpha \neq \alpha_{0}$. Then, taking the inner product of (106) with $g$ and using the fact that $\left\langle g, f_{0}\right\rangle=-1 / \alpha_{0}$, we have $\langle g, f\rangle=0$. Hence, by (106) again, $f=0$. Thus (100) follows.

Let $\alpha=\alpha_{0}$. Then

$$
A f_{0}=v=-\alpha_{0}\left\langle g, f_{0}\right\rangle v .
$$

Hence $f_{0} \in \operatorname{ker} A\left(\alpha_{0}\right)$. Thus, by (107), we obtain (101).

(ii) We have

$$
A(\alpha)^{*}=A^{*}+\alpha^{*}\langle v, \cdot\rangle g .
$$

Hence, by replacing $\{A, g, v, \alpha\}$ by $\left\{A^{*}, v, g, \alpha^{*}\right\}$ in the proof of (i), we can easily obtain (102).

(iii) For all $f \in \operatorname{ker} A(\alpha)$, we have (105). Let $\alpha \neq 0$. Then (105) means that $\langle g, f\rangle v \in D\left(A^{-1}\right)$. Hence, if $v \notin\left(A^{-1}\right)$, then $\langle g, f\rangle=0$, which implies via (105) again that $A f=0$; that is, $f=0$. Thus (103) follows.

If $v \in D\left(A^{-1}\right)$ with $\left\langle g, A^{-1} v\right\rangle=0$, then $f=-\alpha\langle g, f\rangle A^{-1} v$ and hence $\langle g, f\rangle=0$. Hence $f=0$. Thus (103) follows.

(iv) It is similar to the proof of (iii).

Theorem 23. (i) Assume (C.1). Then

$$
\begin{aligned}
& \operatorname{ker} Q\left(\alpha_{0}\right) \\
& \quad=\oplus_{n, p=0}^{\infty}\left[\left(\otimes^{n}\left\{z A^{-1} v \mid z \in \mathbb{C}\right\}\right) \otimes \wedge^{p}\left(\operatorname{ker} A\left(\alpha_{0}\right)^{*}\right)\right],
\end{aligned}
$$

and hence $\operatorname{nul} Q\left(\alpha_{0}\right)=\infty$.
Moreover, for all $\alpha \neq \alpha_{0}$,

$$
\operatorname{ker} Q(\alpha)=\oplus_{p=0}^{\infty} \mathbb{C} \otimes \wedge^{p}\left(\operatorname{ker} A(\alpha)^{*}\right) .
$$

(ii) Assume (C.2). Then

$$
\begin{aligned}
& \operatorname{ker} Q\left(\beta_{0}\right) \\
& =\oplus_{n=0}^{\infty}\left\{\left[\otimes_{s}^{n} \operatorname{ker}\left(A\left(\beta_{0}\right)\right)\right] \otimes\left[\mathbb{C} \oplus \mathscr{L}\left(\left\{A^{*-1} g\right\}\right)\right]\right\}, \\
& \operatorname{ker} Q(\alpha)=\oplus_{n=0}^{\infty}\left[\otimes_{s}^{n} \operatorname{ker} A(\alpha) \otimes \mathbb{C}\right], \quad \alpha \neq \beta_{0} .
\end{aligned}
$$

(iii) Assume (C.3). Then, for all $\alpha \in \mathbb{C}$,

$$
\operatorname{ker} Q(\alpha)=\oplus_{p=0}^{\infty}\left[\mathbb{C} \otimes \wedge^{p}\left(\operatorname{ker} A(\alpha)^{*}\right)\right] .
$$

(iv) Assume (C.4). Then, for all $\alpha \in \mathbb{C}$,

$$
\operatorname{ker} Q(\alpha)=\oplus_{n=0}^{\infty}\left[\otimes_{s}^{n} \operatorname{ker} A(\alpha) \otimes \mathbb{C}\right] .
$$

Proof. Under condition (C.1), we have (100) and (101). Hence $\otimes_{s}^{n} \operatorname{ker} A\left(\alpha_{0}\right)=\otimes^{n}\left\{z A^{-1} v \mid z \in \mathbb{C}\right\}$. Therefore, applying (77) and Theorem 4 , we obtain (110).

In the case $\alpha \neq \alpha_{0}$, (111) follows from (100), (77), and Theorem 4.

Using Lemma 22, one can prove (ii)-(iv) in the same manner as in the proof of (i).

Some compatible compositions of conditions (C.1)-(C.4) yield the following results.

Corollary 24. (i) Assume (C.1) and (C.2). Then

$\operatorname{ker} Q\left(\alpha_{0}\right)$

$$
\begin{gathered}
=\overline{\mathscr{L}\left(\left\{a\left(A^{-1} v\right)^{* n} \Omega_{b} \otimes b\left(A^{*-1} g\right)^{* j} \Omega_{f} \mid n \geq 0, j=0,1\right\}\right),} \\
\operatorname{ker} Q(\alpha)=\left\{c \Omega_{b} \otimes \Omega_{f} \mid c \in \mathbb{C}\right\}, \quad \alpha \neq \alpha_{0} .
\end{gathered}
$$

(ii) Assume (C.1) and (C.4). Then

$$
\begin{gathered}
\operatorname{ker} Q\left(\alpha_{0}\right)=\overline{\mathscr{L}\left(\left\{a\left(A^{-1} v\right)^{* n} \Omega_{b} \otimes \Omega_{f} \mid n \geq 0\right\}\right),} \\
\operatorname{ker} Q(\alpha)=\left\{c \Omega_{b} \otimes \Omega_{f} \mid c \in \mathbb{C}\right\}, \quad \alpha \neq \alpha_{0} .
\end{gathered}
$$

(iii) Assume (C.2) and (C.3). Then

$$
\begin{gathered}
\operatorname{ker} Q\left(\beta_{0}\right)=\mathscr{L}\left(\left\{\Omega_{b} \otimes b\left(A^{*-1} g\right)^{* j} \Omega_{f} \mid j=0,1\right\}\right) . \\
\operatorname{ker} Q(\alpha)=\left\{c \Omega_{b} \otimes \Omega_{f} \mid c \in \mathbb{C}\right\}, \quad \alpha \neq \beta_{0} .
\end{gathered}
$$

(iv) Assume (C.3) and (C.4). Then, for all $\alpha \in \mathbb{C}$,

$$
\operatorname{ker} Q(\alpha)=\left\{c \Omega_{b} \otimes \Omega_{f} \mid c \in \mathbb{C}\right\} .
$$




\section{Fredholm Property and the Index $\operatorname{ind}_{\Gamma} Q(\alpha)$}

Theorem 23 has also implications for Fredholm property of $Q(\alpha)$. Since we want to see how Fredholm property of $Q_{A}$ may change under the perturbation $V_{g, v}(\alpha)$, we take our consideration under the same conditions as in Theorem 5.

We first note the following fact.

Lemma 25. Let $A$ be semi-Fredholm. Then, for all $\alpha \in \mathbb{C}, A(\alpha)$ is semi-Fredholm and

$$
\text { ind } A=\text { ind } A(\alpha) \text {. }
$$

Proof. We have (71) and $T_{g, v}$ is a one-rank operator, which is compact. Thus, by a general theorem [12, Chapter IV, Theorem 5.26], the lemma holds.

Theorem 26. (i) Suppose that (C.1) holds and A is Fredholm. Then $Q\left(\alpha_{0}\right)_{+}$is not semi-Fredholm and, for all $\alpha \in \mathbb{C} \backslash\left\{\alpha_{0}\right\}$, $\mathrm{Q}(\alpha)_{+}$is Fredholm with

$$
\operatorname{ind}_{\Gamma} Q(\alpha)=\delta_{0, \text { ind } A^{*}}=\operatorname{ind}_{\Gamma} Q_{A} .
$$

(ii) Suppose that (C.3) holds and $A$ is Fredholm. Then, for all $\alpha \in \mathbb{C}, Q(\alpha)$ is Fredholm and (120) holds.

(iii) Suppose that (C.2) holds and A is semi-Fredholm. Then $Q\left(\beta_{0}\right)_{+}$is not semi-Fredholm and, for all $\alpha \in \mathbb{C} \backslash\left\{\beta_{0}\right\}$, the following hold.

(a) If $A$ is injective, then $Q(\alpha)_{+}$is Fredholm with $\operatorname{ind}_{\Gamma} Q(\alpha)=1$.

(b) If $A$ is not injective, then $Q(\alpha)_{+}$is semi-Fredholm with $\operatorname{ind}_{\Gamma} Q(\alpha)=\infty$.

(iv) Suppose that (C.4) holds and that A is semi-Fredholm.

(a) If $A$ is injective, then, for all $\alpha \in \mathbb{C}, Q(\alpha)_{+}$is Fredholm and $\operatorname{ind}_{\Gamma} Q(\alpha)=1$.

(b) If $A$ is not injective, then, for all $\alpha \in \mathbb{C}, Q(\alpha)_{+}$is semi-Fredholm and $\operatorname{ind}_{\Gamma} Q(\alpha)=\infty$.

(v) Let $A$ be semi-Fredholm with nul $A \geq 1$ and $\operatorname{nul} A^{*} \geq$ 1. Suppose that $g \in(\operatorname{ker} A)^{\perp}$ and $v \in\left(\operatorname{ker} A^{*}\right)^{\perp}$. Then $Q(\alpha)_{+}$ is not semi-Fredholm.

Proof. In the proofs below, (77) is used without mentioning explicitly.

(i) By Lemma 22-(i), nul $A\left(\alpha_{0}\right)=1$. By this fact and Lemma 25 , we have

$$
\operatorname{nul} A\left(\alpha_{0}\right)^{*}=1+\operatorname{nul} A^{*} \geq 1 .
$$

Hence, applying Theorem 5-(iii) with $A$ replaced by $A\left(\alpha_{0}\right)$, one concludes that $Q\left(\alpha_{0}\right)_{+}$is not semi-Fredholm.

Let $\alpha \neq \alpha_{0}$. Then, by Lemma 25, we have nul $A^{*}=$ nul $A(\alpha)^{*}$. Hence, by Theorem 5-(i), $Q(\alpha)_{+}$is Fredholm and $\operatorname{ind}_{\Gamma} Q(\alpha)=\delta_{0, \text { nul } A(\alpha)^{*}}=\delta_{0, \text { nul } A^{*}}$. Thus (120) holds.

(ii) It is similar to (i).

(iii) In the present case, we have nul $A^{*}=0$ and $\operatorname{nul} A\left(\beta_{0}\right)^{*}=1$. Hence, by Lemma $25, \operatorname{nul} A\left(\beta_{0}\right)=\operatorname{nul} A+1 \geq$ 1 . Hence, by applying Theorem 5-(iii) with $A$ replaced by $A\left(\beta_{0}\right)$, we conclude that $Q\left(\beta_{0}\right)_{+}$is not semi-Fredholm.

Let $\alpha \neq \beta_{0}$. Then, by Lemma 22-(ii), $A(\alpha)^{*}$ is injective. By Lemma 25, nul $A=\operatorname{nul} A(\alpha)$. (a) Let $A$ be injective. Then $A(\alpha)$ is injective. Hence, we can apply Theorem 5-(i) with $A$ replaced by $A(\alpha)$ to conclude that $Q(\alpha)_{+}$is Fredholm with $\operatorname{ind}_{\Gamma} Q(\alpha)=$ 1.

(b) Let $A$ be not injective. Then nul $A(\alpha) \geq 1$. Hence, we can apply Theorem 5-(ii) with $A$ replaced by $A(\alpha)$ to conclude that $Q(\alpha)_{+}$is semi-Fredholm with ind ${ }_{\Gamma} Q(\alpha)=\infty$.

(iv) In the present case, we have nul $A(\alpha)^{*}=0$ for all $\alpha \in \mathbb{C}$. Hence, by Lemma $25, \operatorname{nul} A=\operatorname{nul} A(\alpha)$ for all $\alpha \in \mathbb{C}$.

(a) In this case, nul $A(\alpha)=0$. Hence, by Theorem 5-

(i) with $A$ replaced by $A(\alpha), Q(\alpha)$ is Fredholm and $\operatorname{ind}_{\Gamma} Q(\alpha)_{+}=1$.

(b) In this case, we can apply Theorem 5-(ii) with $A$ replaced by $A(\alpha)$ to conclude that $Q(\alpha)_{+}$is semiFredholm and $\operatorname{ind}_{\Gamma} Q(\alpha)_{+}=\infty$.

(v) In the present case, we have $\operatorname{ker} A \subset \operatorname{ker} A(\alpha)$ and $\operatorname{ker} A^{*} \subset \operatorname{ker} A(\alpha)^{*}$. Hence nul $A(\alpha) \geq 1$ and nul $A(\alpha)^{*} \geq 1$. Therefore, we can apply Theorem 5-(iii) with $A$ replaced by $A(\alpha)$ to conclude that $Q(\alpha)_{+}$is not semi-Fredholm.

\section{Nonzero Eigenvalues of $Q(\alpha)$}

Finally we consider existence of nonzero eigenvalues of $Q(\alpha)$. In this section we need the following condition.

Hypothesis (I). (i) $\mathscr{H}=\mathscr{K}$;

(ii) $A$ is an injective and nonnegative self-adjoint operator;

(iii) $g=v \in D\left(A^{-1}\right)$.

Under Hypothesis (I), the constant $\alpha_{0}$ defined by (98) takes the form

$$
\alpha_{0}=-\frac{1}{\left\langle v, A^{-1} v\right\rangle}<0 .
$$

Theorem 27. Let Hypothesis (I) be satisfied and $\alpha<\alpha_{0}(<0)$. Then, there exists a unique constant $x_{0}(\alpha)<0$ such that $\alpha\left\langle v,\left(x_{0}(\alpha)-A\right)^{-1} v\right\rangle=1$ and, for all $n \in\{0\} \cup \mathbb{N}$,

$$
\pm \sqrt{n} x_{0}(\alpha) \in \sigma_{p}(Q(\alpha)) .
$$

with eigenvectors

$$
\begin{gathered}
{\left[Q(\alpha) \pm \sqrt{n} x_{0}(\alpha)\right]\left\{a\left(\phi_{\alpha}\right)^{* n-p} \Omega_{b} \otimes b\left(\phi_{\alpha}\right)^{* p} \Omega_{f}\right\}} \\
\quad \in \operatorname{ker}\left(Q(\alpha) \mp \sqrt{n} x_{0}(\alpha)\right) \quad(n \geq p \geq 0),
\end{gathered}
$$

where

$$
\phi_{\alpha}:=\left(x_{0}(\alpha)-A\right)^{-1} v .
$$

Moreover, $x_{0}(\alpha)$, as a function of $\alpha<\alpha_{0}$, is strictly monotone increasing on $\left(-\infty, \alpha_{0}\right)$ with $\lim _{\alpha \rightarrow-\infty} x_{0}(\alpha)=-\infty$ and $\lim _{\alpha \rightarrow \alpha_{0}} x_{0}(\alpha)=0$.

Note that Theorem 27 holds even if $Q_{A}$ has no nonzero eigenvalues. This is an interesting phenomenon. Since the 
condition $\alpha<\alpha_{0}<0$ implies that $|\alpha|>\left|\alpha_{0}\right|$, the phenomenon may be regarded as a strong coupling effect.

To prove Theorem 27, we prepare two lemmas.

We consider a condition slightly more general than Hypothesis (I).

Hypothesis (II). In addition to (i) and (ii) in Hypothesis (I), the following holds: $v \in D\left(A^{a}\right) \cap D\left(A^{-1}\right)$ for some $a \in \mathbb{R}$ and

$$
g=A^{a} v
$$

Under Hypothesis (II), the constant $\alpha_{0}$ defined by (98) takes the form

$$
\alpha_{0}=-\frac{1}{\left\langle A^{a} v, A^{-1} v\right\rangle}<0 .
$$

Lemma 28. Let Hypothesis (II) be satisfied.

(i) Let $\alpha_{0}<\alpha$. Then there exist no solutions $x \leq 0$ to the equation

$$
\alpha\left\langle A^{a} v,(x-A)^{-1} v\right\rangle=1 .
$$

(ii) Let $\alpha \leq \alpha_{0}(<0)$. Then there exists a unique constant $x_{0}(\alpha) \leq 0$ such that

$$
\alpha\left\langle A^{a} v,\left(x_{0}(\alpha)-A\right)^{-1} v\right\rangle=1 .
$$

The constant $x_{0}(\alpha)$ is, as a function of $\alpha \leq \alpha_{0}(<$ $0)$, strictly monotone increasing and differentiable on $\left(-\infty, \alpha_{0}\right)$ with $x_{0}\left(\alpha_{0}\right)=0$ and $\lim _{\alpha \rightarrow-\infty} x_{0}(\alpha)=-\infty$.

Proof. (i) Since $A$ is injective, nonnegative, and self-adjoint, we can define a function $F$ on $(-\infty, 0]$ by

$$
F(x):=-\left\langle A^{a} v,(x-A)^{-1} v\right\rangle>0, \quad x \in(-\infty, 0] .
$$

This function is differentiable on $(-\infty, 0)$ with

$$
\frac{d F(x)}{d x}=\left\langle A^{a} v,(x-A)^{-2} v\right\rangle>0, \quad x \in(-\infty, 0) .
$$

Hence $F$ is strictly monotone increasing. It is easy to see that $\lim _{x \rightarrow-\infty} F(x)=0$ and $\lim _{x \rightarrow 0} F(x)=-1 / \alpha_{0}>0$ (note that $\left.\alpha_{0}<0\right)$. Hence, if $\alpha>\alpha_{0}$, then $-\alpha F(x)=1$ has no solutions $x$ in $(-\infty, 0]$.

(ii) Let $\alpha \leq \alpha_{0}$. Then, by the proof of (i), there exists a unique constant $x_{0}(\alpha) \leq 0$ such that $-\alpha F\left(x_{0}(\alpha)\right)=1$. The inverse function $F^{-1}$ of $F$ exists on $\left(0,-1 / \alpha_{0}\right]$ and is differentiable on $\left(0,-1 / \alpha_{0}\right)$. Hence $x_{0}(\alpha)=F^{-1}(-1 / \alpha), \alpha \leq$ $\alpha_{0}$. This implies the assertion on $x_{0}(\alpha)$.

Lemma 29. Let Hypothesis (II) be satisfied.

(i) Let $\alpha>\alpha_{0}$. Then

$$
\sigma_{p}(A(\alpha)) \cap(-\infty, 0]=\emptyset .
$$

(ii) Let $\alpha \leq \alpha_{0}$ and $\phi_{\alpha}$ be defined by (125). Then

$$
\begin{gathered}
\sigma_{p}(A(\alpha)) \cap(-\infty, 0]=\left\{x_{0}(\alpha)\right\}, \\
\operatorname{ker}\left(A(\alpha)-x_{0}(\alpha)\right)=\left\{c \phi_{\alpha} \mid c \in \mathbb{C}\right\} .
\end{gathered}
$$

Proof. Let $x \in \sigma_{p}(A(\alpha)) \cap(-\infty, 0]$. Then there is a nonzero vector $\phi \in D(A)$ such that $A \phi+\alpha\left\langle A^{a} v, \phi\right\rangle v=x \phi$. It follows that

$$
\phi=\alpha\left\langle A^{a} v, \phi\right\rangle(x-A)^{-1} v .
$$

If $\left\langle A^{a} v, \phi\right\rangle=0$, then $\phi=0$, which is a contradiction. Hence $\left\langle A^{a} v, \phi\right\rangle \neq 0$. Taking the inner product of (135) with $A^{a} v$, we obtain

$$
\alpha\left\langle A^{a} v,(x-A)^{-1} v\right\rangle=1 .
$$

(i) If $\alpha>\alpha_{0}$, then, by Lemma 28-(i), (136) has no solutions $x$ in $(-\infty, 0]$. Hence (132) holds.

(ii) Let $\alpha \leq \alpha_{0}$. Then, by Lemma 28-(ii), we have $x=$ $x_{0}(\alpha)$. Thus $\sigma_{p}(A(\alpha)) \cap(-\infty, 0] \subset\left\{x_{0}(\alpha)\right\}$. It is easy to check that $A(\alpha) \phi_{\alpha}=x_{0}(\alpha) \phi_{\alpha}$. Thus (133) follows. By (135), every vector $\phi \in \operatorname{ker}\left(A(\alpha)-x_{0}(\alpha)\right)$ satisfies $\phi=\alpha\left\langle A^{a} v, \phi\right\rangle \phi_{\alpha}$. Hence $\phi \in\left\{c \phi_{\alpha} \mid c \in \mathbb{C}\right\}$. Thus (134) follows.

Proof of Theorem 27. Under the present assumption, $A(\alpha)$ is self-adjoint. Hence, by Lemma $29, x_{0}(\alpha)^{2} \in \sigma_{p}\left(A(\alpha)^{2}\right)$. Hence Theorem 16 implies (123). We have $A(\alpha)^{2} \phi_{\alpha}=$ $x_{0}(\alpha)^{2} \phi_{\alpha}$. Hence

$$
\Phi^{(n, p)}(\alpha):=a\left(\phi_{\alpha}\right)^{* n-p} \Omega_{b} \otimes b\left(\phi_{\alpha}\right)^{* p} \Omega_{f} \quad(n \geq p \geq 0)
$$

are eigenvectgors of $Q_{A(\alpha)}^{2}=Q(\alpha)^{2}$ with eigenvalue $n x_{0}(\alpha)^{2}$. Hence, applying Lemma 8 with $S=Q(\alpha)$, we see that $(Q(\alpha) \pm$ $\left.\sqrt{n} x_{0}(\alpha)\right) \Phi^{(n, p)}(\alpha) \in \operatorname{ker}\left(Q(\alpha) \mp \sqrt{n} x_{0}(\alpha)\right)$.

\section{Conflict of Interests}

The author declares that there is no conflict of interests regarding the publication of this paper.

\section{Acknowledgment}

This work is supported by the Grant-In-Aid no. 24540154 from JSPS.

\section{References}

[1] A. Arai, "A general class of infinite-dimensional Dirac operators and path integral representation of their index," Journal of Functional Analysis, vol. 105, no. 2, pp. 342-408, 1992.

[2] A. Arai, "Path integral representation of the index of KählerDirac operators on an infinite-dimensional manifold," Journal of Functional Analysis, vol. 82, no. 2, pp. 330-369, 1989.

[3] A. Arai, "Dirac operators in Boson-Fermion Fock spaces and supersymmetric quantum field theory," Journal of Geometry and Physics, vol. 11, no. 1-4, pp. 465-490, 1993.

[4] A. Arai, "Supersymmetric extension of quantum scalar field theories," in Quantum and Non-Commutative Analysis, H. Araki, Ed., pp. 73-90, Kluwer Academic Publishers, Dordrecht, The Netherlands, 1993.

[5] A. Arai, "Supersymmetric quantum field theory and infinitedimensional analysis," Sugaku Expositions, vol. 9, no. 1, pp. 8798, 1996. 
[6] A. Jaffe and A. Lesniewski, "Supersymmetric quantum fields and infinite dimensional analysis," in Nonperturbative Quantum Field Theory, G. Hooft, A. Jaffe, G. Mack, P. K. Mitter, and R. Stora, Eds., Plenum, New York, NY, USA, 1988.

[7] A. Arai, "On self-adjointness of Dirac operators in bosonfermion Fock spaces," Hokkaido Mathematical Journal, vol. 23, no. 2, pp. 319-353, 1994.

[8] A. Arai, Fock Spaces and Quantum Fields, Nippon Hyoronsha, Tokyo, Japan, 2000 (Japanese).

[9] O. Bratteli and D. W. Robinson, Operator Algebras and Quantum Statistical Mechanics 2, Springer, Berlin, Germany, 2nd edition, 1997.

[10] M. Reed and B. Simon, Methods of Modern Mathematical Physics Vol.II: Fourier Analysis, Self-Adjointness, Academic Press, New York, NY, USA, 1975.

[11] M. Reed and B. Simon, Methods of Modern Mathematical Physics. I. Functional Analysis, Academic Press, New York, NY, USA, 1972.

[12] T. Kato, Perturbation Theory for Linear Operators, Springer, Berlin, Germany, 2nd edition, 1976.

[13] S. Futakuchi and K. Usui, "On the spectra of fermionic second quantization operators," http://arxiv.org/abs/1306.3001.

[14] K. Schmüdgen, Unbounded Self-Adjoint Operators on Hilbert Space, Springer, Dordrecht, The Netherlands, 2012.

[15] P. A. Deift, "Applications of a commutation formula," Duke Mathematical Journal, vol. 45, no. 2, pp. 267-310, 1978.

[16] M. Reed and B. Simon, Methods of Modern Mathematical Physics : Analysis of Operators, vol. 4, Academic Press, New York, NY, USA, 1978.

[17] A. Arai, "Perturbation of embedded eigenvalues: a general class of exactly soluble models in Fock spaces," Hokkaido Mathematical Journal, vol. 19, no. 1, pp. 1-34, 1990. 


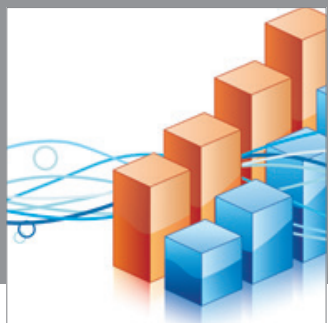

Advances in

Operations Research

mansans

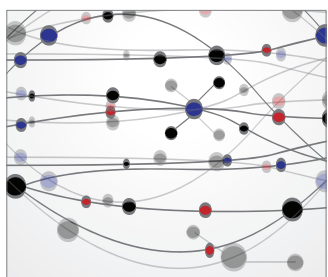

The Scientific World Journal
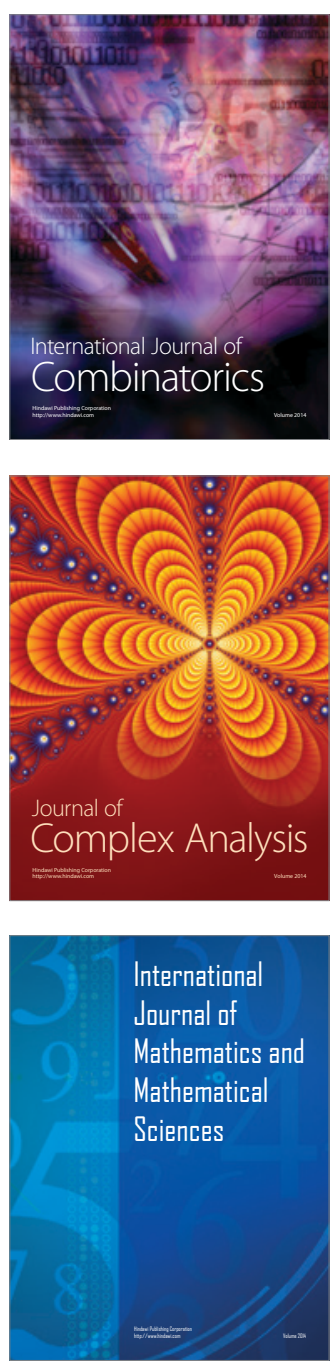
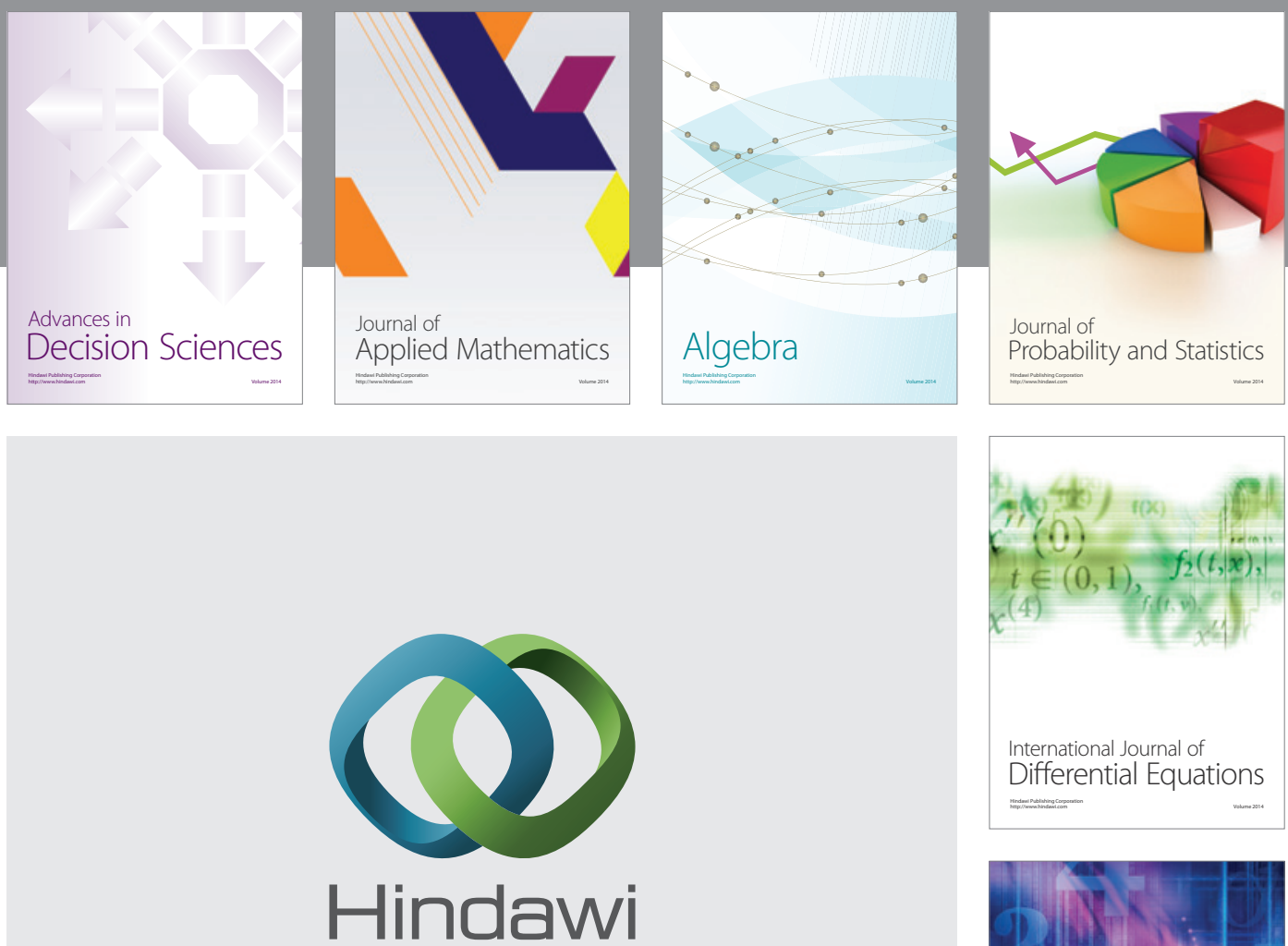

Submit your manuscripts at http://www.hindawi.com
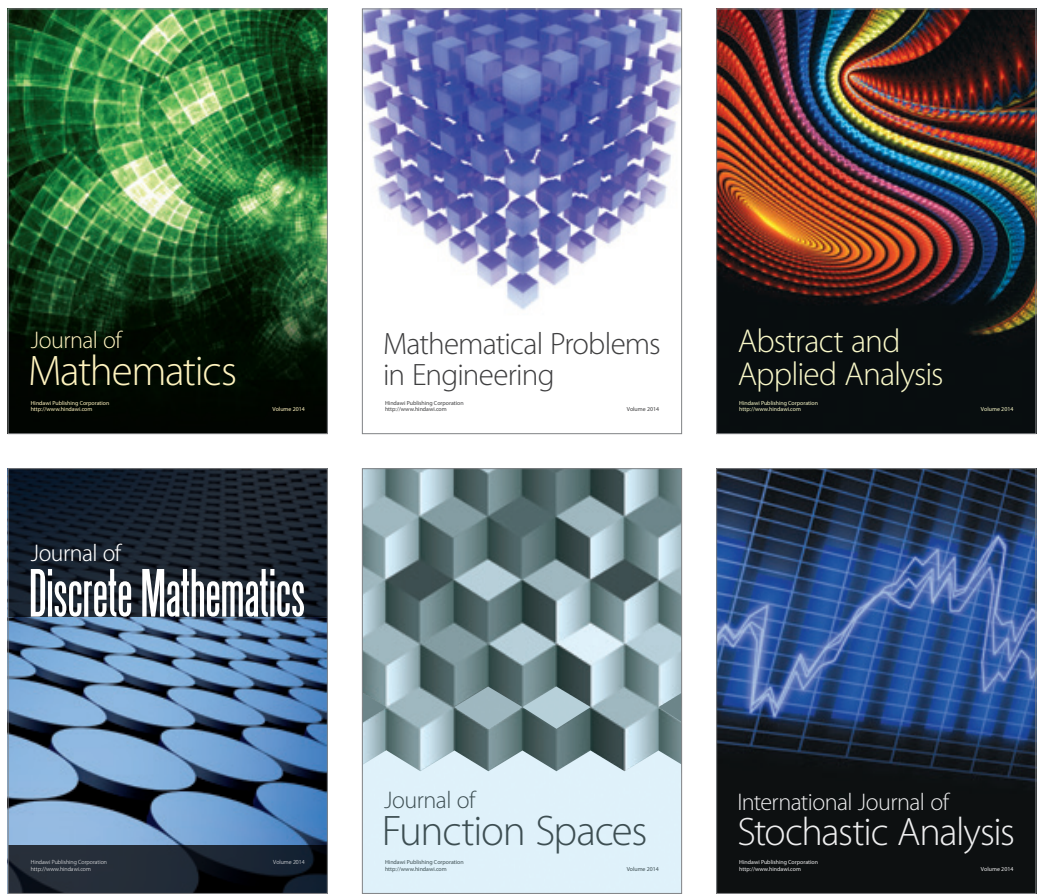

Journal of

Function Spaces

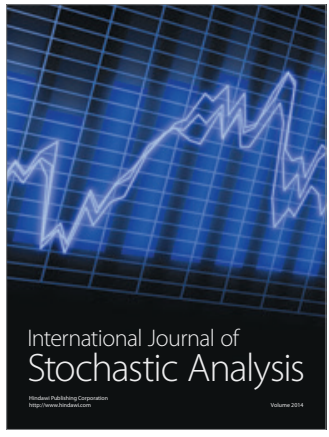

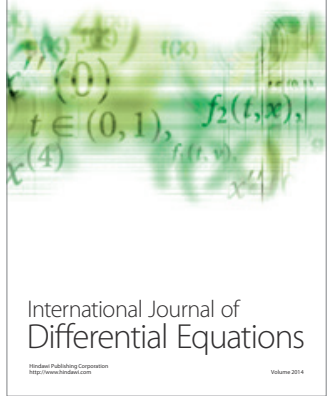
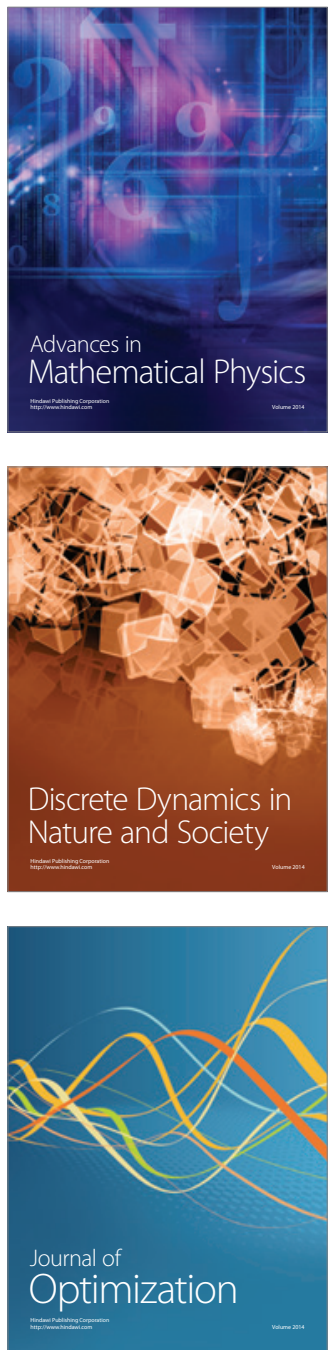